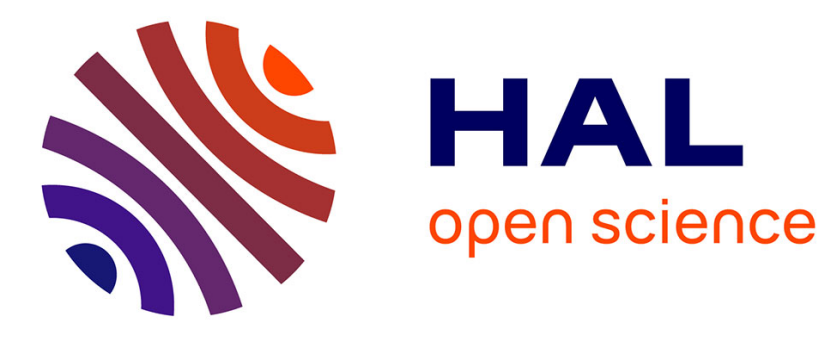

\title{
Construction and assessment of a 3-T MRI brain template.
}

Florent Lalys, Claire Haegelen, Jean-Christophe Ferré, Omar El-Ganaoui, Pierre Jannin

\section{- To cite this version:}

Florent Lalys, Claire Haegelen, Jean-Christophe Ferré, Omar El-Ganaoui, Pierre Jannin. Construction and assessment of a 3-T MRI brain template.. NeuroImage, 2010, 49 (1), pp.345-54. 10.1016/j.neuroimage.2009.08.007 . inserm-00546487

\section{HAL Id: inserm-00546487 https://www.hal.inserm.fr/inserm-00546487}

Submitted on 14 Dec 2010

HAL is a multi-disciplinary open access archive for the deposit and dissemination of scientific research documents, whether they are published or not. The documents may come from teaching and research institutions in France or abroad, or from public or private research centers.
L'archive ouverte pluridisciplinaire HAL, est destinée au dépôt et à la diffusion de documents scientifiques de niveau recherche, publiés ou non, émanant des établissements d'enseignement et de recherche français ou étrangers, des laboratoires publics ou privés. 


\section{Construction and assessment of a 3T MRI brain template}

Florent Lalys ${ }^{1,23}$, Claire Haegelen ${ }^{1,23,4}$, Jean-Christophe Ferre ${ }^{1,23,5}$, Omar ElGanaoui $^{1,23}$, Pierre Jannin ${ }^{1,23}$

1

INSERM, U746, Faculté de Médecine CS 34317, F-35043 Rennes Cedex, France

2 INRIA, VisAGeS Unité/Projet, F-35042 Rennes, France

${ }^{3}$ University of Rennes I, CNRS, UMR 6074, IRISA, F-35042 Rennes, France

${ }^{4}$ Department of Neurosurgery, Pontchaillou University Hospital, F-35043 Rennes, France

5 Department of Radiology, Pontchaillou University Hospital, F-35043 Rennes, France

Corresponding author:

Florent Lalys (florent.lalys@irisa.fr)

Unite / Equipe U746 VISAGES

IRISA

Campus de Beaulieu

35042 Rennes CEDEX

France

Tel.: +33 (2) 23233829

Fax: +33 (2) 99847171 


\title{
Keywords
}

Brain Template, basal ganglia, 3T MRI, Deep Brain Stimulation

Word count:

4055

Abstract word count:

164

Tables:

Figures:

References:

8

44

\begin{abstract}
New MR imaging protocols enable visualisation of brain structures. However, for dedicated clinical applications such as targeting Deep Brain Stimulation (DBS), a more accurate localisation requires the use of atlases. We developed a three-dimensional digitised mono-subject anatomical template of the human brain based on 3T MR images. By averaging 15 registered T1 image acquisitions, we have shown that the final image corresponds to an optimal image, limited by the performance of the 3T MR machine. We compared different preprocessing workflows for template construction. With the optimal strategy along with validated existing processing methods, one $\mathrm{T} 1$ template, one $\mathrm{T} 2$ template and one T1-T2 mixing template were created in order to improve visualisation of spatially complex deep structures. Reduction of voxel size to $0.25 \mathrm{~mm}^{3}$ was also advantageous to observe fine structures and White Matter/Gray Matter intensity crossings. Results demonstrated that such a template also improved inter-patient registration for population comparison in DBS. These MR templates are made freely available to our community (http://www.vmip.org/mritemplate) to serve as a reference for neuro-image processing methods.
\end{abstract}




\section{Introduction}

The development of medical imaging equipment is driving increased demand for reference data sets. Anatomical reference images are becoming of vital importance for comparison of results, to achieve optimal spatial and intensity resolution, and to allow better identification of structures. One of them are the templates, which are defined as anatomical models built from multiple volumes averaging. They can be mono or multi-subjects and are generally based on a single modality. Atlases are derived from templates but can be built from different modalities and are most often characterised by specific structures labelling. Paper-based atlases were originally obtained from experts in anatomy who manually drew and labelled reference images. For instance, atlases of the human brain turn out to be very helpful for various procedures. Print atlases by Schaltenbrand and Wahren (1977), Talairach and Tourmoux (1988), or Ono et al (1990) have been used with success in various computer aided decision systems. Today, digital atlases are directly built from digital images and offer new capabilities and applications. Nowinski et al (1997) used a combination of these three digitized print atlases to develop a digital atlas. They are used as anatomy teaching tools, by providing interactive labelling of structures and high images resolution and contrast. In image analysis, deformable atlases provide a powerful tool for image segmentation, by exploiting constraints derived from the image data together with a priori knowledge about structure parameters (Zhou and Rajapakse, 2005). Elastic registrations to a standard atlas of the organ of interest are also very useful, enhancing objectivity of image interpretation (Friston et al., 1995). Finally, they may serve as a common space for population comparisons studies, such as Voxel-Based Morphometry (VBM), which allows identification of anatomical differences between groups of subjects (Shen et al., 2005).

Digital templates can be classified according to the number of subjects used for the computation. Multi-subject templates are mainly built from single acquisition of different control subjects reflecting the population targeted by the clinical study (Seghers et al., 2004; Lee et al., 2005). Such multi-subject templates are primarily intended to serve as anatomical references for spatial normalisation usually required before studying human anatomical or functional variability. A prominent example is the 
multimodal template used in SPM (Statistical Parametric Mapping, Institute of Neurology, University College of London, UK; Evans et al., 1993). The construction of smoothed multiple subject templates captures inter-subject variability, but their use for alignment could hinder the representation of targets in a common space.

Single subject templates strive to attain optimal spatial resolution. Different strategies were followed for defining such templates. The first strategy was the use of co-registered mono or multimodal acquisitions of a single particular subject mainly based on MR acquisitions. The Colin27 MRI brain template (Holmes et al., 1998) has been used in various neurosurgical applications. For instance, StJean et al. (1998) created a deformable volumetric template of the basal ganglia and thalamus in combination with the Schaltenbrand and Wahren atlas in order to estimate template-to-patient transformations. It has also been used to create probabilistic functional templates, as the one by Finnis et al (2003) for the combination of intraoperative data with MRI data, or to validate template warping techniques (Chakravarty et al., 2008). The second strategy was the combination of images and histological templates (Yelnik et al., 2007; Chakravarty et al., 2006). The interest of the first strategy is that the whole clinical acquisition setup is applied to healthy, living control subjects and they prove to be quite feasible in practice. Histological templates have advantages like higher structural and spatial resolutions. However they turn out to be very complicated and lengthy to produce. Finally, templates following the first strategy are based on the average of all registered volumes, which enhances the quality of the final template by increasing Signal-to-Noise Ratio (SNR) and contrast (essentially between Gray Matter (GM) and White Matter (WM)). These templates are constrained by both the resolution and the quality of available imaging technologies.

Digital templates need to be evaluated and deeply validated as they are used for anatomical reference images in various applications. It's crucial to assess the quality of the images by quantifying their parameters, from the contrast resolution to the Signal-to-Noise Ratio (SNR). Many parameters step in for the creation of a template, including the MR machine intrinsic settings, the number of scans to be averaged, the pre-processing methods, the order of pre-processing, and the processing parameters. All 
of these parameters have to be optimized in order to create the template with the optimal construction strategy. The Colin27 MRI brain template has been lightly validated in the original work of Holmes et al., (1998) by computing intensity profiles to demonstrate the improvement in image quality, but no such studies have been performed in depth. Moreover, templates have to be validated in a clinical context to assess its actual added value. In neurosurgery, they are widely used for surgical planning and targeting, especially in DBS (Deep Brain Stimulation). DBS is a procedure for patients with movement disorders (e.g., Parkinson's disease) for which medical therapy is not effective. It uses electrical impulses to stimulate targets (often the SubTalamic Nucleus, STN) in the brain. For such neurosurgical procedures, identification of basal ganglia on patient specific images isn't always possible due to the lack of contrast between structures. The use of digital atlases has helped for addressing this problem as deep brain structures are more visible and allow more accurate targeting.

We present in this paper how we built and assessed MRI templates using a one-subject average of volumes acquired with off-the-shelf medical image protocols, and processed with up-to-date image processing methods. The objective is to create one T1 template with an optimal construction strategy that we will validate in this paper. This framework relies on the fact that the quality of the template increases with the number of volumes averaged, as demonstrated by Holmes et al. (1998), on condition that every volume is perfectly defined in the same common space. The quality reaches a maximum that would theoretically correspond to an MRI without noise and intensity inhomogeneities. We chose to use image processing methods that have already been validated in medical imaging context along with fixed MR machine parameters. However, we evaluated the impact of the order of image preprocessing, the number of scan required to reach an optimal image and the global evolution of the image quality. We showed quantitative results using different complementary criteria demonstrating the effectiveness of the best strategy. Then, we studied the impact of template choice in a nonlinear registration task in a Deep Brain Stimulation (DBS) context. Finally, with the optimal strategy we constructed a $\mathrm{T} 2$ template in order to create a multi-modal brain template by mixing $\mathrm{T} 1$ and $\mathrm{T} 2$ weighted data for better visualisation of deep brain structures. The basic principle was to retain helpful information from T2, i.e. deep brain structures (such as basal ganglia), and to merge this Region Of 
Interest (ROI) with the remaining T1 MRI area. The T1 template aims at helping patient-to-template image registration applied in neurosurgical procedures, whereas the T1-T2 mixed template aims at identifying basal ganglia.

\section{Material and Methods}

\section{Image acquisition}

The subject was a 45 year-old man without any clinical pathology. Absence of brain pathology was checked by a neuroradiologist on the two first sets of MR images. Fifteen T1-weighted sequences and 7 T2-weighted sequences were performed on a Philips Achieva 3T system (Philips Medical Systems, Best, The Netherlands) using an 8-channel head coil, on the dates in Table 1. For the selected T1weighted sequence, the parameters were: 3D Fast Field Echo Sequence, sagittal acquisition, 160 continuous slices, section thickness $=1 \mathrm{~mm}$, field of view $=256 \mathrm{~mm}, \mathrm{TR} / \mathrm{TE} / \mathrm{TI}=9.8 / 4.6 / 915 \mathrm{~ms}$, flip angle $=8^{\circ}$, SENSE factor $=1.5$, matrix $=256 \times 256$ (acquisition), $512 \times 512$ (after zero-fill interpolation), voxel size $=0.5 \times 0.5 \times 1 \mathrm{~mm}$, and acquisition time $=5 \mathrm{~min} .05 \mathrm{~s}$. For the selected $2 \mathrm{D}$ T2-Weighted sequence, the parameters were: 2D Turbo Spin Echo Sequence, coronal acquisition, 64 continuous slices, section thickness $=1 \mathrm{~mm}$, field of view $=256 \mathrm{~mm}, \mathrm{TR} / \mathrm{TE}=3035 / 80 \mathrm{~ms}$, flip angle $=90^{\circ}$, Echo train length $=15$, matrix $=256 \times 256$, voxel size $=1 \times 1 \times 1 \mathrm{~mm}$, and acquisition time $=7 \mathrm{~min} .17 \mathrm{~s}$.

There was minimal patient motion between acquisitions within the same session, except for the eighth where the subject got out of the machine between the two scans in order to simulate two different sessions. In each session, a first Talairach repositioning was performed before launching scans to fit the $\mathrm{x}$-axis with AC-PC. Each session took place between 1PM and 2PM, just after lunch, endeavouring to keep the same physiological patient conditions. DICOM images were stored on dedicated CDs.

\section{Preprocessing}


All T1 and T2-weighted MR images were first denoised with the non-local means algorithm (Coupé et al., 2008), which has been successfully validated on 3T MR images (Coupé et al., 2006). A bias correction algorithm based on intensity values (Mangin, 2000) was then applied to all images using BrainVisa software (CEA, Orsay, France, http://brainvisa.info), which was well adapted for 3T MR images (Vovk et al., 2007). An intensity-normalisation step was not necessary since all images were acquired in accordance with the same protocol, on the same subject.

\section{Construction of the templates}

Registration: One T1 native image was randomly chosen to be the target for registration. We realigned the target volume to have the AC-PC line on y-axis and the mid-sagittal plane on the z-axis. All volumes ( $\mathrm{T} 1$ and $\mathrm{T} 2$ ) were then linearly registered to the target (rigid registration, cost function: Mutual Information, NEWUOA optimisation (Wiest-Daesslé et al., 2007)). Every native image was defined in the same common space, which allowed us to perform the average.

Average: We used a method called kappa-sigma clipping average (Jorsater, 2006). This method, largely used in astronomy, finds and deletes pixels that are significantly different from the median. Median and ${ }^{\sigma}$ were first computed for each voxel of the image. A threshold was then applied to reject all the voxels with an intensity superior to mediar $\stackrel{\square}{-}$ - $a p p \otimes \sigma$. This method helped reduce the influence of artefacts, which are caused by the patient or external factors, e.g, scanner table vibrations (Bernstein et al., 2004). Values of kappa of two or three are usually used in the literature (. Moreover, internal studies have shown that the quality of the template was unchanged from a value of kappa of 1.8. We chose this value and rejected approximately $2 \%$ of all voxels and possible outliers.

Subsampling: Resulting averaged volumes were subsampled, using a cubic B-Spline algorithm. For the $\mathrm{T} 1$ template, a two-fold reduction was computed for the $\mathrm{x}$ and $\mathrm{y}$-axes, and a three-fold reduction for the z-axis. For the T2 template, a four-fold reduction was computed for all axes, so that we obtained a $0.25 \mathrm{~mm}^{3}$ voxel volume size for both templates. 
T1+T2 fusion: T2-weighted images provide information on deep brain structures that are not visible in T1-weighted images. In order to take advantage of both MR protocols, we computed a mixed T1+T2 template as follows. We first inversed the intensity of the T2 images in order to fit the WM/GM crossings and the global contrast of the T1 images. An expert defined a bounding box containing basal ganglia. Image intensities from T2 images were kept inside the bounding box; the remaining signal was derived from the T1 images. The final step included boundary smoothing. We deliberately applied a low level of smoothing to reveal the boundaries between both modalities.

\section{Effectiveness of the strategy}

In order to perform a first visual assessment on the contrast quality, we used the intensity profile. This non-quantitative criterion was extracted from the native non-preprocessed image and from the 15volumes average. The section was taken in the coronal planes through WM and GM.

The image quality evaluation studies for the $\mathrm{T} 1$ template were based on five quality criteria that allowed comparing the final 15 -volumes average (used as a reference for the evaluation studies) with the intermediate $\mathrm{N}$-volumes average in order to evaluate and quantify the evolution of improvement. We initially used image intensity correlation $(Q C 1)$ and Mutual Information $(Q C 2)$, defined in the whole brain. These criteria are both based on intensity values and are widely used in medical image processing to evaluate differences between two images. We then used the Signal-to-Noise Ratio (SNR) (QC3), defined by:

$$
S N R_{r}=10 \cdot \log \square \frac{255}{M S E} \square
$$

where $M S E$ is the Mean Square Error between the reference volume (the 15-volumes average image) and the intermediate volume. Another possibility for estimating the SNR was to compute the signal on a Region Of Interest (ROI) divided by the standard deviation of the background noise, which is usually defined outside the anatomy on a MR image. This one, after pre-processing and a few volume 
averages, was null, which made impossible to perform a global study until the 15 -volumes average image. For this reason, we preferred using the first formula for the estimation of the SNR.

Another vital quality parameter is the contrast, essentially between WM and GM. Similarly to SNR, the usual contrast equation (Fushimi et al., 2007) supposes to compute the standard deviation of the background noise, so it is not applicable to our studies. To quantify it, we computed the acutance (QC4) (Choong et al., 2003), which is the edge contrast of an image:

$$
A C=\frac{G}{x_{G M}-x_{W M}}
$$

where ${ }^{G}$ is the mean density gradient between GM and WM, and $X_{G M}-X_{W M}$ the distance between GM and WM regions. It represents the amplitude of the derivative of brightness with respect to space, and approaches the definition of the sharpness of the image. Additionally, the gradient term adds a notion of distance that is essential in the concept of contrast. A simple $C d=\frac{S_{G M}-S_{W M}}{D}$ contrast difference $(Q C 5)$ was also computed, where $D$ is the dynamic range of the image, $S_{G M}$ is the signal in the GM region and $S_{W M}$ the signal in the WM region. For the computation of the contrast (acutance and contrast), GM and WM regions of frontal lobes and basal ganglia (putamen, thalamus, etc.) were selected as ROIs by a neuroradiologist. A total of $10 \mathrm{WM} / \mathrm{GM}$ intensity crossings were chosen in order to obtain an overall representative contrast. In each average step, the same ROIs were applied.

To study the impact of preprocessing, we performed the same studies for: 1) the native images without preprocessing, 2) images with denoising only, 3) images with bias correction only, 4) images with bias correction followed by denoising and 5) images with denoising followed by bias correction. The average was computed with the volumes in the same order as their acquisitions. All volumes were warped on the reference target with the transformations found in Construction of the templates Registration.

We performed two final studies using correlation criterion to assess the impact of the order of images in template construction. The first study compared the average of five images from the same session with five from different sessions. For this study, the evaluation reference was the final 5-volumes 
average. Similarly, the second study compared a randomised selection of acquisitions with the temporal ordered selection.

Intra-subject registration validation: As the quality of the final template was dependent on the quality of the intra-subject image registration, we studied all native $\mathrm{T} 1$ registrations with the $\mathrm{T} 1$ target. Nine anatomical landmarks were defined by a neurosurgeon and identified on each floating and reference image. Five of them (Figure I) were defined within the bounding box describe in the "construction of the templates" subsection: the Anterior and Posterior Commissures (CA and CP, Point 1 and 3 respectively), the interthalamic adhesion on the middle of the axial slice (point 2), the infundibular recesse (Point 4), and the middle between the mamillary bodies on the axial slice just above the last visualisation of the optic chiasma (Point 5). The four others are the left and right carotid division into anterior and middle cerebral arteries, and the middle of the origin of the trigeminal nerve (also left and right). The Euclidean distance for each landmark defined in both images was computed, in order to obtain the global misplacement error. A similar study was performed to assess the T2 to T1 registration.

\section{Validation for clinical studies}

We studied the impact on patient-to-template image registration of our final template compared with the improved version of the Colin27 template (Aubert-Broche et al., 2006). We applied the same registration workflow to a set of 15 patients with idiopathic Parkinson's diseases (Lalys et al., 2009). The pre-operative T1 MR images of each patient were used. All subjects had subthalamic nucleus (STN) DBS according to selected inclusion criteria (Lang and Lozano, 1998; The DBS for Parkinson's Disease Study Group, 2001). Exams were performed on a 3T whole-body imager (Achieva, Philips Medical Systems, Best, The Netherlands) by using a transmit-receive head coil and were acquired with a Fast Field Echo sequence after injection of Gadolinium. The acquisition parameters were as follows: $\mathrm{TE} / \mathrm{TR} / \mathrm{Flip}$ angle $=4.6 \mathrm{msec} / 9.9 \mathrm{msec} / 8^{\circ}$, acquired matrix size $=256 \times 256 \mathrm{~mm}$, field of view $(\mathrm{FOV})=$ $256 \mathrm{~mm}$, voxel size $=1 \times 1 \times 1 \mathrm{~mm}$, volume $=182$ axial $1 \mathrm{~mm}$ thickness slices, no SENSE factor, and acquisition time $=6 \min 59$. The $\mathrm{T} 1$ template was resampled to fit the Colin27 template $\left(0.5 \mathrm{~mm}^{3}\right.$ voxel 
volume size). We used a strategy close to the one described by Sanchez et al. (2006), which states that the best registration of patient images to the atlas in the DBS context was a global affine image to atlas registration, followed by a non-linear registration using a Demons algorithm along with semiautomated segmentations of deep structures. The registration procedure proposed here has been adapted to be fully automated and to be close to the one proposed by Sanchez. The following registration workflow was applied for each patient: affine T1-MR-to-atlas registration was first computed. Then a local affine registration was computed on a region of interest including the deep brain structures. The final step included a nonlinear local registration step using the Demons approach [www.itk.org], which estimated a 3D deformation field between a source volume and a target volume. A landmark-based validation study was applied, similar to the intra-subject registration validation study. As we decided to limit this study to a DBS context, the registration validation was based only on the five anatomical landmarks in the bounding box (Figure I), which were manually defined by a neurosurgeon on the 15 volumes and on the two templates.

\section{Results}

\section{Construction of the templates}

Volume averaging visually enhances the global quality of the template (Figure II). Figure III shows the homogenisation of the signal and an accurate visualisation of GM and WM regions on the T1 template. On the mixed T1-T2 template, the bounding box is visible but intensities of the inverse-T2 globally match the T1 boundaries. This allows visualisation of deep brain structures with high resolution as well as a better demarcation of GM/WM intensity crossings.

Figure IV shows the 7-T2 volumes average before contrast inversion and the subsampling step. Deep brain structures are clearly visible in the mixed T1-T2 template, e.g. the transversal slices on Figure V.

\section{Effectiveness of the strategy}


Intensity profile: Figure VI shows a visual improvement, as GM/WM intensity crossings are more significant in the averaged image. Each GM/WM intensity region is defined in lower/higher intensities, thereby increasing the differentiation between them. We observed a reduction in noise, as WM areas seem to be more homogeneous.

Figures VIIa, VIIb, VIIc, VIId and VIIe show image quality criteria for each N-intermediate average volume. Computations for Figures VIIa, VIIb and VIIc were based on a ground truth: the 15-volumes image.

For all criteria, the five first images (corresponding to acquisitions of the first session) did not have a significant impact on the final result and on the improvement of quality criteria. The profiles increased, but very slowly. When the acquisition session changed, at the fifth average, all criteria were suddenly raised, until the sixth or seventh average. The comments from the first session could also be applied, since from the fifth to tenth averages it corresponded to the second session. The images subjected to a bias correction were superior to those without this pre-processing. In terms of acutance, this result was valuable starting from the sixth average. For correlation, acutance, SNR and contrast, the profiles seemed to reach a maximum, whereas it was not the case for mutual information. We found that an average with images first denoised, and then corrected, had almost the same profile as the average with images first corrected, and then denoised. Moreover, in all criteria, profiles from non-preprocessed images were almost identical to those only denoised.

In Figure VIII, the averages based on acquisitions from different sessions evolve more than the ones based on acquisitions from the same session, which did not vary. In Figure IX, the profile of randomised image averages had a more linear increase than the ordered image averages, without the sudden change at the fifth and tenth average. 
Intra-subject registration: For all $15 \mathrm{~T} 1$ volumes, the global placement error was null; all five anatomical landmarks perfectly matched their corresponding points on the native target. The same results were found with the $\mathrm{T} 2$ registered volumes.

\section{Validation for clinical studies}

We found a placement error of $1.58+/-0.33 \mathrm{~mm}$ for the registration with the Colin 27 template, and $0.78+/-0.19 \mathrm{~mm}$ with our template. Anova was computed for the 15-patient study on Figure $\mathrm{X}$ $(\mathrm{p}<0.001)$.

\section{Discussion}

In the current study, we constructed and validated MR mono-subject brain templates. This kind of template could serve as a teaching tool to visualise complex deep structures and as a spatial reference for neurological studies. Different criteria were studied, first in order to find the optimal strategy for building this template, and then to demonstrate the impact of using such high quality image templates in patient-to-template registration. Results demonstrated the quality of the resulting template and its impact on accurate registration.

\section{Influence of $3 \mathrm{~T}$ machine}

It has recently been proven that signal-to-noise ratio is significantly better at $3 \mathrm{~T}$ than at $1.5 \mathrm{~T} \mathrm{MR}$ imaging (Hoenig et al., 2005; Manka et al., 2005; Frayne et al., 2003; Schick, 2005; Yongbi et al., 2002). Other authors (Nobauer-Huhmann et al., 2002, Scarabino et al., 2003; Sasaki et al., 2003; Ross, 2004) have reported lower contrast between GM and WM at 3T, but it was subjective, as it was based 
on visual assessment only. In (Fushimi et al., 2007), differences of contrast between 1.5 and 3T were quantitatively computed with the CNR and best results were shown for the $3 \mathrm{~T}$ images.

\section{Patient motion}

As mentioned earlier, the ideal result would be an image without noise, inhomogeneities and partial volume effects, keeping in mind that the major source of improvement is due to the SNR increase. As already mentioned (Holmes et al., 1998), a small patient motion between each acquisition seems to be essential to increase the quality of the final template. It can be explained by the modification of the magnetic fields and the noise inside the machine. Intrinsic machine parameters do not significantly change between consecutive acquisitions. That means that averaging these images is comparable to averaging virtually identical images. Figures VIII and IX show that it is essential to acquire scans from different sessions in order to decrease the noise. To rapidly raise the quality of our template, starting from the tenth acquisitions, we chose to wait a few days between each scan. With this approach, we were assured that the subject did not have the same physical location in the machine, and that intrinsic machine parameters had fluctuated. Moreover, it allows us to suggest that the intense signal regions of the magnetic field could have moved between acquisitions and that the global spatial energy could be reformed after the average. As we can see in Figure VII, for almost all criteria the global quality of the volumes does not significantly increase from the 12th to 13th average. This allows us to suggest that the template had reached a limit corresponding to an optimal image without noise and with a global contrast corresponding to the threshold of $3 \mathrm{~T}$ machine performances.

\section{Registration validation}

With current imaging technologies, intra-subject rigid registration appears to be very effective, especially when native acquisitions are subject to a reliable preprocessing step. A good spatial definition of the volumes in a common space is required to precisely compute the average. The main drawback of this method is a deterioration of the contrast with higher GM/WM intensity crossings. As we found no registration error, the quality of the final template is not distorted by inaccurate intrasubject registration. 


\section{Preprocessing influence}

As already mentioned, the preprocessing workflow was divided into two principal components: inhomogeneity correction (IC) and noise correction using a Non Local Means (NLM) method. Without loss of generality, we have chosen the reference image as the image pre-processed by (NLM+IC).

In Figures VIIa, VIIb and VIIc, profiles that did not include a preprocessing step of IC were always higher than those including this step. This can be explained by the fact that the image histogram was significantly changed by the IC, and was therefore more influential on intensity values than a simple NLM. This argument also explains why the two profiles with NLM preprocessing alone and without preprocessing are relatively similar in all Figures. In Figures VIId and VIIe, profiles that did not include a preprocessing step of IC were above those including this step until the sixth acquisition. This is probably due to the dynamic spread that decreases the contrast and the acutance, which is closely related to the contrast definition.

The order of the two preprocessing steps is often related to the application (Montillo et al., 2003) and can modify the results of the image processing applied after these treatments. For Figures VIIa, VIIb, VIIc and VIIe, the profiles NLM+IC and IC+NLM are nearly identical. Only Figure VIId shows a significant difference between the two profiles, with IC+NLM higher than NLM+IC. These observations fit the previous assumption that the preprocessing has to comply with the image processing. When noise removal is applied first, the weak edges can be smoothed and will not be recovered by inhomogeneity correction. For image processing dedicated to segmentation, the IC is usually used before denoising, which is often unnecessary to obtain good segmentation (Pham and Prince, 1999). In our case, the order of the two operations has no consequences. Indeed, acutance is the edge contrast of an image, and is therefore different from simple contrast and from the three other criteria.

IC may remove some anatomical information but we were not interested in recovering the exact bias field, just a delineation of the tissue's gray level distribution (Mangin, 2000). 


\section{Usefulness of T1-T2 mixed template}

Multi-subject MR templates have been shown to allow better visual inspection of deep brain structures due to better contrast, since averaging improved the signal-to-noise ratio (D'Haese et al., 2005; Bardinet et al., 2008). Mono-subject MR templates further increase contrast since there is no anatomical variability between scans. Our T1-T2 mixed template further improves contrast of basal ganglia, such as the subthalamic nucleus and the globus pallidus, which are targets of DBS in patients with movement disorder. Our templates would be useful in the preoperative workflow for improved DBS targeting (Stancanello et al., 2008; Dawant et al., 2007; Guo et al., 2005), as well for postoperative assessment (Lalys et al., 2009). Such high-resolution low-noise templates could also be used to evaluate and improve atlas-based image processing methods, such as segmentation (Scherrer el al., 2008; Pohl et al., 2006).

Results of the comparative patient-to-template image registration study have also shown that the $\mathrm{T} 1$ template was a good reference for registration methods. Finally, these templates could serve as a teaching tool to visualise complex structures, which are barely visible in current MR images.

\section{Validation for clinical studies}

Results of the patient-to-template registration comparison have shown that our T1 template increases the accuracy of a patient-to-template registration procedure. Recent study on patients with Parkinson's diseases (Rocha Vasconcellos et al., 2009) has proved that no significant anatomical differences exist for these patients when compared to a control group. However, limitations of this validation study can be found in the limitation of such landmark-based approach. One uncertainty concerns the spatial localisation of anatomical landmark which is limited by the image resolution. In order not to bias the landmark-based validation, we performed on our T1 template a resampling step to have the same image resolutions for both templates. The major limitation was the number of experts and subjects used. Even if significant differences between both templates were found, a larger study is required to completely validate the contribution of our new template, with for instance inter and intra-rater variability computation. Nevertheless, the reduced placement error can be due to the high field MR images used 
for the study. Our $3 \mathrm{~T}$ template seems to be more able to serve as anatomical reference when images have high quality.

\section{Conclusion}

In this paper, we have reported on the construction of in vivo healthy human neuroanatomy MR templates. Our objective was to optimise spatial and intensity resolutions. The reduction of noise enhanced the visibility of fine structures, as both contrast and SNR increased with the number of volumes averaged. Such image quality is not available with current imaging protocols. Small movements between scans turned out to be a vital condition to enhance the quality of the final template. We demonstrated that the three computed templates allow visualisation of spatially complex structures as well as increased contrast between GM and WM. We also showed that they greatly improved the accuracy of template based registration. Both resulting templates are freely available online (http://www.vmip.org/mritemplate). 


\section{References}

Aubert-Broche B, Evans AC, Collins L, 2006. A new improved version of the realistic digital brain phantom. NeuroImage, 32, 138-145.

Bardinet E, Bhattacharjee M, Dormont D, Pidoux B, Malandain G, Schüpbach M, Ayache N, Cornu P, Agid Y, Yelnik J, 2008. A three-dimensional histological atlas of the human basal ganglia. II. Atlas deformation strategy and evaluation in deep brain stimulation for Parkinson disease. J Neurosurg, 31, $1-12$.

Bernstein MA, King KF, Zhou XJ, 2004. Handbook of MRI pulse sequence. Elsevier, Amsterdam.

Chakravarty M, Bertrand G, Hodge CP, Sadikot AF, Collins DL, 2006. The creation of a brain atlas for image guided neurosurgery using serial histological data. NeuroImage, 30(2), 359-376.

Chakravarty M, Sadikot AF, Germann J, Bertrand G, Collins DL, 2008. Towards a validation of atlas warping techniques. Medical Image Analysis, 12, 713-726.

Choong YF, Rakebrandt F, Morgan JE, 2003. Acutance, an objective measure of retinal nerve fibre image clarity. British J of Ophthalmology, 87, 322-326.

Clouchoux C, Coulon O, Anton J, Mangin J, Regis J, 2006. A new cortical surface parcellation model and its automatic implementation. MICCAI'06, International Conference on Medical Image Computing and Computer Assisted Intervention, in Lecture Notes in Computer Science, Springer, Copenhaguen, Denmark, 4191, 193-200.

Coupé P, Yger P, Barillot C, 2006. Fast Non Local Means Denoising for 3D MR Images. MICCAI'06, International Conference on Medical Image Computing and Computer Assisted Intervention, in Lecture Notes in Computer Science, Springer, Copenhaguen, Denmark, 4191, 33-40.

Coupé P, Yger P, Prima S, Hellier P, Kervram C, Barillot C, 2008. An Optimized Blockwise Non Local Means Denoising Filter for 3D Magnetic Resonance Images. IEEE Trans. on Medical Imaging, 24(4), 425-441.

D’Haese PF, Cetinkaya E, Konrad PE, Kao C and Dawant MB, 2005. Computer-aided placement of deep brain stimulators: from planning to intraoperative guidance. IEEE Trans. on Medical Imaging, 24(11), $1469-1478$. 
Dawant B, D’Haese P-F, Pallavaram S, Li R, Yu H, Spooner J, Davis T, Kao C, Konrad P, 2007. The VUDBS project: integrated and computer-assisted planning, intra-operative placement, and post-operative programming of deep-brain stimulators. Proceedings of SPIE: Medical Imaging, San Diego, CA, 6509, 701-710.

Evans AC, Collins DL, Mills SR, Brown ED, Kelly RL, Peters TM, 1993. 3D statistical neuroanatomical models from 305 MRI volumes, 1993. Proc IEEE Nucl Science Symp Medl Imaging Conf, 1813-1820.

Finnis KW, Starreveld YP, Parrent AG, Sadikot AF, Peters TM, 2003. Three-dimensional database of subcortical electrophysiology for image-guided stereotactic functionnal neurosurgery. IEEE Trans. on Medical Imaging, 22(1), 93-104.

Franz B, Diego C, Deborah L, Daniele N, 2003. The Description Logic Handbook: Theory, Implementation, and Applications. Cambridge University Press.

Frayne R, Goodyear BG, Dickhoff P, Lauzon ML, Sevick RJ, 2003. Magnetic resonance imaging at 3.0 Tesla: challenges and advantages in clinical neurological imaging. Invest Radiol, 38, 385-402.

Friston KJ, Holmes AP, Worsley KJ, Poline JP, Frith CD, Frackowiak RSJ, 1995. Statistical parametric maps in functional imaging. Human Brain Mapping, 2, 189-210.

Fushimi Y, Miki Y, Urayama S, Okada T, Mori N, Hanakawa T, Fukuyama H, Togashi K, 2007. Gray matter-white matter contrast on spin-echo T1-weighted images at $3 \mathrm{~T}$ and $1.5 \mathrm{~T}$ : a quantitative comparison study. European Radiology, 17(11), 2921-2925.

Guo T, Finnis KW, Parrent AG, Peters TM, 2005. Development and Application of Functional Databases for Planning Deep-Brain Neurosurgical Procedures. MICCAI’05, International Conference on Medical Image Computing and Computer Assisted Intervention, in Lecture Notes in Computer Science, Springer, Palm Springs, USA, 3749, 835-842.

Guo T, Parrent AG, Peters TM, 2007. Surgical targeting accuracy analysis of six methods for subthalamic nucleus deep brain stimulation. Comp Aid Surg, 12(6), 325-334.

Hoenig K, Kuhl CK, Scheef L, 2005. Functional 3.0-T MR assessment of higher cognitive function: are there advantages over 1.5-T imaging? Radiology, 234, 860-868.

Holmes CJ, Hoge R, Colin L, Woods R, Toga AW, Evans AC, 1998. Enhancement of MR Images Using Registration for Signal Averaging. J Comput Assist Tomogr, 22(2), 324-333. 
Hunter JJ, Cookson CJ, Kisilak ML, Bueno JM, Campbell MC, 2007. Characterizing image quality in a scanning laser ophthalmoscope with differing pinholes and induced scattered light. Journal of the Optical Society of America, 24(5), 1284-1295.

Jorsater S, 2006. Methods in astronomical image processing with special applications to the reduction of CCD data. Lecture Notes in Physics, 413, 193-207.

Lalys F, Haegelen C, Abadie A, Jannin P, 2009. Post-operative assessment in Deep Brain Stimulation based on Multimodal images: Registration Workflow and Validation. Proceedings of SPIE: Medical Imaging, Orlando, FL, 7261.

Lang AE, Lozano AM, 1998. Parkinson's Disease. First of two Parts. The New England Journal of medicine, 339(15), 1044-1053.

Lee JS, Lee DS, Kim J, Kim YK, Kang E, Kang H, Kang KW, Lee JM, Kim JJ, Park HJ, Kwon JS, Kim SI, Yoo TW, Chang KH, Lee MC, 2005. Development of Korean Standard Brain templates. J Korean Med Science, 20, 483-461.

Mangin J-F, 2000. Entropy minimization for automatic correction of intensity non uniformity. Hilton Head Island, SC: IEEE Press, 162-169.

Manka C, Traber F, Gieseke J, Schild HH, Kuhl CK, 2005. Three-dimensional dynamic susceptibilityweighted perfusion MR imaging at 3.0 T: feasibility and contrast agent dose. Radiology, 234, 869-877.

Montillo, A., Udupa, J.K., Axel, L., Metaxas, D.N., 2003. Interaction between noise suppression and inhomogeneity correction in MRI. Proceedings of SPIE: Medical Imaging, San Diego, CA, 5032, 1025-1036.

Newberg HJ, Richards GT, Richmond M, Fan X, 1999. Catalog of Four-Color Photometry of Stars, Galaxies, and QSOS Using SDSS Filters. The Astrophysical J Supl. Series, 123(2), 377-435.

Nobauer-Huhmann IM, Ba-Ssalamah A, Mlynarik V, Barth M, Schoggl A, Heimberger K, Matula C, Kaider A, Trattnig S, 2002. Magnetic resonance imaging contrast enhancement of brain tumors at 3 Tesla versus 1.5 Tesla. Invest Radiol, 37, 114-119.

Nowinski WL, Fang A, Nguyen BT, Raphel JK, Jagannathan L, Raghavan R, Bryan NR, Miller GA, 1997. Multiple brain atlas database and atlas based neuroimaging system. Computer Aided Surgery, 2(1), 4266. 
Ono M, Kubik S, Abernathy CD, 1990. Atlas of the cerebral sulci. Georg Thieme Verlag/Thieme medical publishers, Stuttgart, Germany.

Pham, D.L., Prince, J.L, 1999. An adaptive fuzzy C-means algorithm for image segmentation in the presence of intensity inhomogeneities. Pattern Recognit Letters, 20(1), 57-68.

Pohl KM, Fisher J, Grimson WE, Kikinis R, Wells WM, 2006. A Bayesian model for joint segmentation and registration. NeuroImage, 31, 228-239.

Rocha Vasconcellos LF, Pereira Novis SA, Moreira DM, Rosso ALZ, ACCB Leite, 2009. Neuroimaging in Parkinsonism: a study with magnetic resonance and spectroscopy as tools in the differential diagnosis. Arq. Neuro-Psiquiatr, 67(1).

Ross JS, 2004. The high-field-strength curmudgeon. AMJN Am J Neuroradiol, 25, 168-169.

Sanchez Castro F, Pollo C, Meuli R, Maeder P, Culsenaire O, Bach Cuadra M, Villemure J, Thiran J, 2006. A Cross Validation Study of Deep Brain Stimulation Targeting: From Experts to Atlas-Based, Segmentation-Based and Automatic Registration. IEEE Trans. on Medical Imaging, 25(11), 14401450.

Sasaki M, Inoue T, Tohyama K, Oikawa H, Ehara S, Ogawa A, 2003. High-field MRI of the central nervous system: current approaches to clinical and microscopic imaging. Magn Reson Med Sci, 2, 133139.

Scarabino T, Nemore F, Giannatempo GM, Bertolino A, Di Salle F, Salvolini U, 2003. 3.0 T magnetic resonance in neuroradiology. Eur J Radiol, 48, 154-164.

Schaltenbrand G, Wahren W, 1977. Atlas for Stereotaxy of the Human Brain. Thieme, Stuttgart, Germany.

Scherrer B, Forbes F, Garbay C, Dojat M, 2008. Fully Bayesian joint model for MR brain tissue and structure segmentation. MICCAI'08, International Conference on Medical Image Computing and Computer Assisted Intervention, in Lecture Notes in Computer Science, Springer, New York, USA, 5242, 1066-1074.

Schick F, 2005. Whole-body MRI at high field: technical limits and clinical potential. Eur Radiol, 15, 946959.

Shen S, Sterr A, Szameitat A, 2005. A template effect study on voxel-based morphometry in statistical parametric mapping. IEEE Eng. In Medicine and Biology, 3051-3054. 
Seghers D, D'Agostino E, Maes F, Vandermeulen D, Suetens P, 2004. Construction of a Brain Template from MR Images Using State-of-the-Art Registration and Segmentation Techniques. Lecture Notes in Computer Science, 3216, 696-703.

St-Jean P, Sadikot AF, Collins L, Clonda D, Kasrai R, Evans AC, Peters TM, 1998. Automated atlas integration and interactive three-dimensional visualization tools for planning and guidance in funcionnal neurosurgery. IEEE Trans. on Medical Imaging, 17(5), 672- 680.

Stancanello J, Muacevic A, Sebastiano F, Modugno N, Cerveri P, Ferrigno G, Uggeri F, Romanelli P, 2008. 3T MRI evaluation of the accuracy of atlas-based subthalamic nucleus identification. Med Phys, 35(7), 3069-77.

Talairach J, Tournoux P, 1988. Co-Planar Stereotaxic Atlas of the Human Brain. Thieme, Stuttgart, Germany.

The Deep-Brain Stimulation for Parkinson's Disease Study Group, 2001. Deep-Brain Stimulation of the Subthalamic Nucleus or the Pars Interna of the Globus Pallidus in Parkinson's Disease. N Engl J Med, 345(13), 956-963.

Von Falkenhausen M, Gieseke J, Morakkabati N, Lutterbey G, Bloehmer G, Kuhl CK, Schild HH, 2004. 3T MRI of the liver after SPIO application. A comparison to 1.5T. Proc Int Soc Magn Reson Med, 11, 904.

Vovk U, Pernus F, Likar B, 2007. A Review of Methods for Correction of Intensity Inhomogneity in MRI. IEEE Trans. on Medical Imaging, 26(3), 405-421.

Wiest-Daesslé N, Yger P, Prima S, Barillot C, 2007. Evaluation of a new optimization algorithm for rigid registration of MRI data. Proceedings of SPIE: Medical Imaging, 6512, 601-610.

Yelnik J, Bardinet E, Dormont D, Malandain G, Ourselin S, Tandé D, Karachi C, Ayache N, Cornu P, Agid Y, 2007. A three-dimensional, histological and deformable atlas of the human basal ganglia. I. Atlas construction based on immunohistochemical and MRI data. NeuroImage, 34(2), 618-38.

Yongbi MN, Fera F, Yang Yihong, Frank JA, Duyn JH, 2002. Pulsed Arterial Spin Labelling: Comparison of Multisection Baseline and Functional MR Imaging Perfusion Signal at 1.5 and 3.0 T: Initial Results in Six Subjects. Radiology, 222, 569-575. 
Zhou J, Rajapakse JC, 2005. Segmentation of subcortical brain structures using fuzzy templates. NeuroImage, 28(4), 915-924. 